\title{
An alternative pathway for preclinical research in fluid management William J Sibbald
}

\author{
Sunnybrook and Women's College Health Sciences Centre, Toronto, Ontario, Canada
}

Received: 3 August 2000

Published: 13 October 2000
Crit Care 2000, 4:S8-S15

(c) Current Science Ltd (Print ISSN 1364-8535; Online ISSN 1466-609X)

\begin{abstract}
Recent meta-analyses have created uncertainties regarding the appropriate clinical role of colloid resuscitation fluids in critically ill patients and prompted changes in fluid management practice. Such changes may not be justified in view of methodological limitations inherent in the meta-analyses. Further research is nevertheless needed to resolve the questions raised concerning the relationship between choice of resuscitation fluid and patient outcome. Animal studies can play an important part by reliably indicating whether particular fluids are likely to prove effective and safe in clinical trials. It is important to avoid costly large-scale clinical trials that fail to demonstrate the clinical utility of the tested therapy, as resources expended in failed trials raise overall development costs and thereby restrict the range of therapies meeting criteria of commercial feasibility. Promising therapies may thus not be pursued, even though an urgent clinical need may exist. An alternative pathway of preclinical research may be of value in avoiding some of the major clinical trial failures of recent years, particularly in the area of sepsis. This alternative pathway commences with the formulation of hypotheses by therapeutics developers. Independent preclinical investigators are challenged, by means of a competitive request for proposals, to test the hypotheses in rigorous randomized studies employing clinically relevant animal models. Promising proposals would then be selected for further development with the aid of peer review. The results of the randomized animal studies, along with other preclinical data, could also be evaluated using accepted principles of 'critical appraisal' commonly applied to clinical trial results. This critical appraisal might, where appropriate, include meta-analysis of animal study findings. This alternative preclinical pathway to new product evaluation should be completed before the commencement of large-scale clinical trials.
\end{abstract}

Keywords: animal disease models, colloids, evidence-based medicine, randomized controlled trials, sepsis, serum albumin

\section{Introduction}

Recent meta-analyses have raised fundamental questions regarding optimal fluid management in critically ill patients. A meta-analysis of randomized, controlled trials comparing colloid with crystalloid administration with respect to mortality did not support continued use of colloids for volume replacement in such patients [1]. Among randomized trials comparing albumin administration with either crystalloid or

$\mathrm{mAb}=$ monoclonal antibody $\mathrm{RFP}=$ request for proposals; TNF = tumour necrosis factor. 
no albumin, a second meta-analysis suggested higher mortality in albumin recipients [2]. Numerous concerns have been voiced regarding the methodological soundness of these meta-analyses. Indeed, a subsequent metaanalysis failed to confirm an increased mortality risk with colloids [3]. Usage of albumin for fluid replacement has nevertheless declined in the wake of these reports [4]. Substitution of artificial colloid for albumin may have accounted for some of this decline.

One limitation of the meta-analyses was inclusion of numerous randomized trials from the late 1970s and early 1980s that may not have accurately reflected current practice. Most of the randomized trials analyzed were, in addition, unblinded, so the possibility of systematic bias cannot be dismissed. It should also be emphasized that meta-analyses with such limitations can be viewed as useful for generating hypotheses rather than supporting evidence-based clinical recommendations [3]. Notwithstanding their limitations, however, the meta-analyses have exerted an impact on clinical practice. Further randomized trials are now, arguably, needed to clarify the clinical utility of colloids, such as albumin. The foundation for such randomized trials should, furthermore, be laid by conducting appropriate preclinical research.

Underscoring the importance of appropriate rigorous preclinical research are the instructive cases of experimental sepsis therapy with monoclonal antibodies (mAb) against tumour necrosis factor (TNF) or endotoxin. These approaches appeared to embody sound therapeutic principles; namely, neutralizing key mediators of sepsis. The animal models employed to evaluate these therapies did not, unfortunately, adequately mimic clinical sepsis [5-7]. The principal preclinical study providing a basis for subsequent clinical trials of anti-TNF mAb therapy involved a baboon bacteraemia model. Baboons received a lethal dose of Escherichia coli, and control animals developed rapid-onset shock and organ failure, succumbing within $7 \mathrm{~h}$ of bacterial challenge. The model was thus based primarily upon intoxication as the relevant pathological process rather than infection, which is more characteristic of clinical sepsis. Subsequent randomized clinical trials failed to demonstrate a survival benefit of anti-TNF mAb therapy $[8,9]$.

The seminal animal study of anti-endotoxin therapy with mAb HA-1A involved, unlike clinical sepsis, pretreatment with the therapeutic agent and neither volume resuscitation nor adjuvant antibiotic therapy [10]. The preclinical evidence was nevertheless judged adequate to justify the initiation of clinical trials. In a double-blind, randomized clinical trial of 543 sepsis patients, no overall difference in survival could be demonstrated, although post hoc subgroup analysis suggested mortality reduction in patients with gram-negative bacteraemia receiving $\mathrm{HA}-1 \mathrm{~A}$ [11]. A second, larger-scale, double-blind, randomized clinical trial was commenced. Prior to publication of its results, however, findings were reported from a randomized study in a canine model of gram-negative septic shock [12]. The canine model was clinically relevant by the criteria that the animals received aggressive fluid resuscitation as well as antibiotics and that $\mathrm{HA}-1 \mathrm{~A}$ was administered subsequent to bacterial challenge; in this model, HA-1A actually decreased survival. The larger-scale clinical trial was reported thereafter. This larger trial, which involved 2199 patients and 603 investigators at 513 community and university-affiliated hospitals in the United States, failed to provide evidence supporting the use of HA-1A for treatment of sepsis [13].

The costly and disappointing failed sepsis clinical trials suggest that there may be more risk-averse and costeffective approaches to be taken in the overall process of preclinical and clinical research by which therapeutics are evaluated. A proposal is presented in this paper that could reduce the overall risks and attendant costs of developing therapeutics for the critical care environment. The proposal calls for an alternative to the conventional pathway for preclinical research (Figure 1). First, developers of therapeutics should assume a leadership role in articulating hypotheses and challenging independent preclinical investigators to design and conduct rigorous randomized studies addressing the hypotheses in animal models that, as faithfully as possible, reproduce the clinical condition of interest. All available preclinical data, including the results of the randomized animal trials, should then be subjected to standard 'critical appraisal', which might include metaanalysis of the available animal studies as appropriate. Initiation of large-scale clinical trials should await the completion of this analysis.

\section{Elements of an alternative pathway}

Animal studies can serve an array of purposes and have conventionally been employed to evaluate the pharmacology, toxicology and mechanisms of action for therapeutics, as well as providing insights into in vivo efficacy and safety (Figure 1). Studies of this type will always continue to play a role in therapeutics development. Animal model studies can and should nevertheless also be designed to yield reliable data addressing specific hypotheses regarding clinical utility for particular indications. The design of animal studies may unfortunately be driven by the research interests of independent preclinical investigators, often using previously established animal models that may not be adequately tailored to mimic accurately a clinical condition of interest. Insufficient emphasis is thus placed on rigorous testing of hypotheses related to clinical utility in this approach. An undesirable byproduct of primarily investigator-driven research is lack of adequate data in the literature bearing on the clinical potential of therapies under development. This byproduct problem could be rectified, 


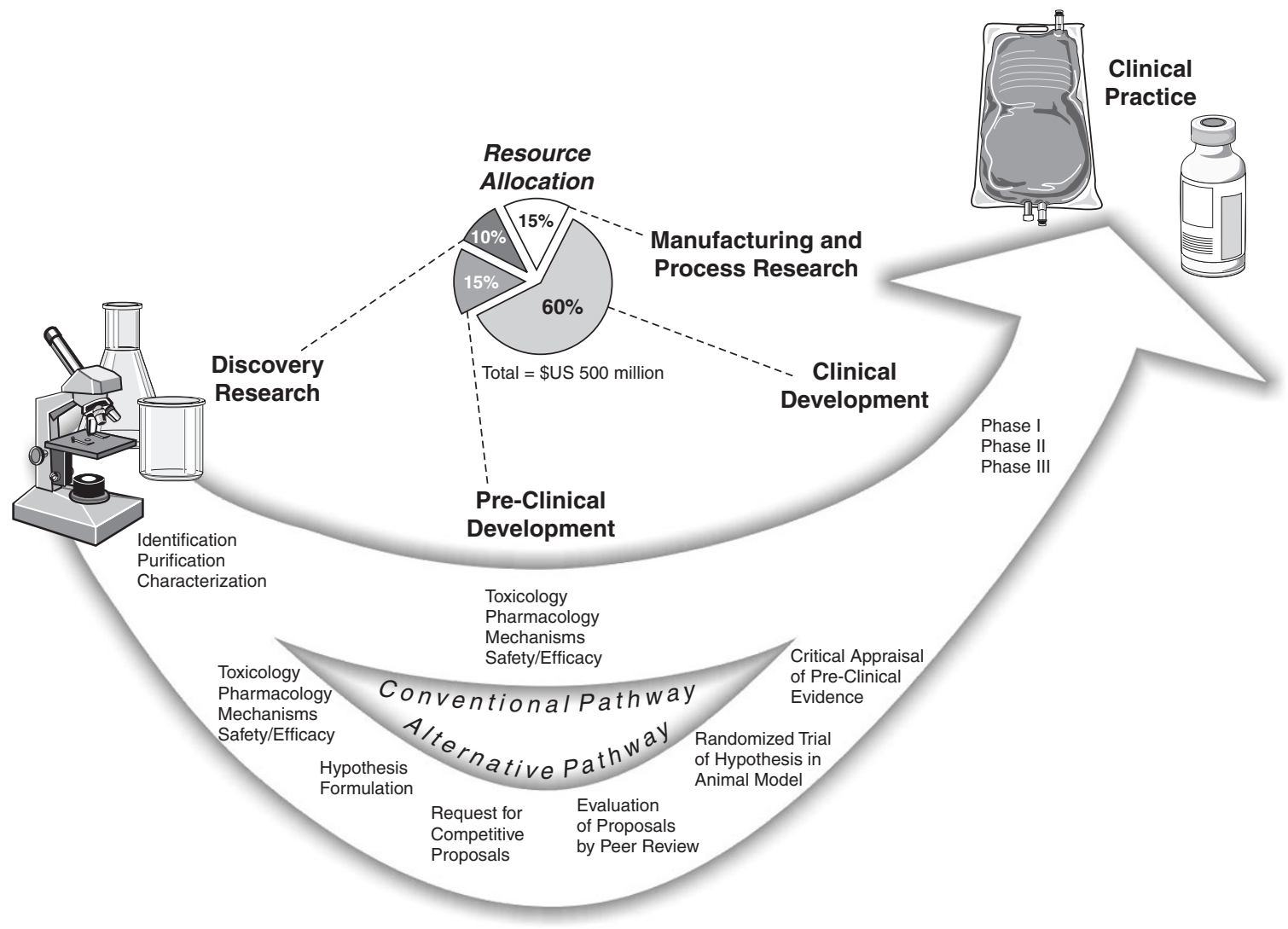

Conventional and proposed alternative pathway for preclinical research, and costs associated with various phases in the development of therapeutics based on the estimates of Drews and Ryser [16].

at least in part, by greater exercise of leadership on the part of therapeutics developers.

These developers should take the responsibility to enunciate the explicit hypotheses that would need to be addressed in determining the worthiness of therapeutics to advance into clinical trials. Therapeutics developers should challenge independent preclinical investigators to design and conduct animal studies that rigorously test these hypotheses, in clinically relevant models. The familiar and well-established 'request for proposals' (RFP) could be used to implement this approach. The RFP would clearly specify the hypotheses to be tested, and would typically appear in medical journals and stipulate a specific time period during which competitive proposals responsive to the RFP might be submitted. Competition in addressing a clearly defined and clinically relevant goal can be expected to elicit the full creativity and resourcefulness of interested preclinical investigators in identifying or devising suitable animal models and designing appropriate studies. This competitive process is likely to culminate in high-quality research proposals. The selection of the best proposals for funding might then be accomplished via peer review, using an advisory panel of experts in the field. The RFP might also allow investigators to propose additional research programme components addressing topics of interest such as mechanisms of action. The needs of the therapeutics developer and the research interests of the independent preclinical investigator might thus be simultaneously accommodated.

A major advantage of this hypothesis-driven competitive research approach is that preclinical investigators will be challenged both to formulate imaginative and sound proposals and, as necessary, to develop improved or novel animal models to replicate the clinical condition of interest. Collaboration with veterinarians may be of value in this regard. Ongoing research makes it clear that animal models can be improved and that novel models can arise, in some instances with the aid of emerging technology. Recently, for example, an improved clinically relevant sepsis model in the conscious rat has been described [7]. Creation of interleukin-10-deficient knockout mice by genetic engineering has additionally provided a new 
Table 1

\begin{tabular}{l} 
Critical appraisal of animal model studies \\
\hline Study selection \\
Indication (eg sepsis) \\
Category \\
Pathophysiology \\
Therapy \\
Other \\
Study assessment \\
Validity of results \\
Randomization \\
Accounting for all experimental subjects \\
Blinding \\
Comparability of groups at baseline \\
Lack of confounders \\
Specific findings \\
Effect size \\
Precision \\
Generalizability to corresponding clinical indication \\
Clinical relevance of animal model \\
Supportive therapies similar to those in the clinical setting \\
Consideration of intercurrent illness \\
Species specificity of the model \\
Potential cost-effectiveness \\
Evidence-based recommendations \\
Strength of evidence \\
Quality of evidence
\end{tabular}

model of intestinal inflammation [14]. It should also be feasible, with the collaboration of veterinarians, to identify models representative of other clinical conditions such as haemorrhagic shock or reperfusion injury.

\section{Critical appraisal of animal model studies}

All available preclinical data, including those generated by the randomized animal model studies, should be subjected to critical appraisal before large-scale clinical trials commence. An approach for critically appraising animal model studies of sepsis has been delineated [15]. This approach is summarized in Table 1 and calls for both the condition of interest, such as sepsis, and the therapeutic intervention to be defined, and proceeds to address a series of key questions. Are the study results valid? Was the study blinded? Was it randomized? What were the results? Were the data subjected to appropriate statistical analysis? Was the study adequately statistically powered to answer the questions being posed? What is the relevance of the animal data to the design and anticipated results of future human studies? Finally, the strength and quality of all the available preclinical evidence need to be taken into account as a basis for recommendations regarding clinical trials to be undertaken.

This type of critical appraisal should be routinely incorporated in the preclinical evaluation of therapeutic modalities, including resuscitation fluids. One obvious benefit would be to avoid misallocation of resources to support unfruitful clinical trials. In this regard, all participants in the healthcare system have a stake in seeing that only clearly promising clinical trials are conducted, since the costs of failed trials will ultimately be born by the overall healthcare system and not solely by the developers of therapies. All participants would, furthermore, benefit from lowering the overall costs associated with developing therapeutics, currently estimated at US\$500 million [16]. This very high typical development cost sharply restricts the range of therapeutics that meet the criteria for commercial feasibility based on standard discounted cash flow rate of return financial models [16]. Numerous promising therapeutics remain undeveloped, notwithstanding clinical need. Rigorous animal studies in clinically relevant models in conjunction with critical appraisal of all available preclinical evidence might, at modest incremental cost, avoid the massive waste of resources entailed in failed large-scale clinical trials and thus contribute to lower overall development costs for therapeutics.

\section{Meta-analysis of animal studies}

One of the important tools for critical appraisal is metaanalysis, which emerged in the $1990 \mathrm{~s}$ as a major approach for fully capitalizing on the wealth of data generated by clinical trials, especially those of relatively small scale. Meta-analysis is a quantitative form of systematic review employing statistical methods to combine and summarize the results of multiple studies [17]. The methodology of meta-analysis is presented in Table 2. Meta-analyses, typically applied to the results of randomized clinical trials, also increasingly address the findings of observational clinical studies [18]. Preclinical data, frequently derived from numerous small-scale animal studies, would also be an appropriate subject for meta-analysis, which might reveal overall trends not evident in small studies affording, individually, only limited statistical power and yielding, collectively, sometimes apparently inconsistent results.

Although animal data have been much less frequently subjected to meta-analysis than clinical data, the feasibility of animal study meta-analysis has been demonstrated in a variety of investigations. For instance, meta-analysis has been reported of rodent studies on dietary fat intake in relation to the occurrence of mammary tumours $[19,20]$. Meta-analytic approaches to animal carcinogenicity experiments have also been described [21,22]. Two meta-analytic investigations have encompassed both animal and human data. In one, separate meta-analyses of 41 rat studies and 17 clinical trials each revealed a significant association between proteinuria and glomerulosclerosis [23]. In the other investigation, separate meta-analyses of four rat and five human studies were conducted to model the relationship between arterial blood toluene levels and behavioural responses [24]. Interestingly, in a metaanalysis on the accuracy of dimercaptosuccinic acid 
scintigraphy for the diagnosis of acute pyelonephritis, it was necessary to focus on animal studies because nephrectomy for histological examination in patients is considered unethical [25].

Animal study meta-analyses to date have underscored the importance of key methodological issues that are also routinely addressed in meta-analyses of human trials. Metaanalyses of animal data have thus included consideration of between-study heterogeneity [26], sensitivity analyses as appropriate [27] and evaluation of included study quality [28].

There are clearly no conceptual barriers to meta-analysis of animal data. Meta-analysis could be routinely conducted as part of the critical appraisal process when multiple suitable animal studies are available. Such meta-analyses would allow more informed decisions as to which therapeutics might advance to clinical trial.

\section{Alternative pathway case studies: fluid management in sepsis}

As one case study exemplifying the proposed alternative pathway for preclinical research, a response to a RFP in the area of fluid management using colloids might characterize the proposed research as follows. First, the goal of this research is to determine if colloid therapy of sepsis ameliorates organ dysfunction, when administered in volumes that do not overwhelm the intravascular space. Second, while mortality is an important outcome for clinical trials of sepsis, organ dysfunction in animal models is an adequate surrogate. Finally, results from this study are to provide information for justification and design of clinical trials.

The proposal might then detail a set of hypotheses to be tested with use of two complementary animal models. It might, for instance, be hypothesized that colloid therapy will: (1) retard the progression of sepsis in sheep, as judged by reduction in tissue injury and gut translocation of endotoxin and improved circulatory reserve to a standardized hypoxic insult; (2) inhibit the progression of sepsis in rats, as evaluated by diminished myocardial, gut and renal dysfunction; and (3) reduce inflammation of the heart associated with sepsis in rats and sheep. Hypotheses (1) and (2) would be intended to satisfy the requirements of the RFP. Hypothesis (3) would address a potential mechanism of colloid action based upon prior evidence, such as the observation that fluid resuscitation can attenuate cytokine gene expression in experimental murine peritonitis [29]. The hypotheses, taken together, would both meet the needs of the RFP sponsor for data to justify and design clinical trials and address special research interests of the investigators submitting the proposal.

In evaluating the research proposal, it would be essential to ask how similar the animal models are to the clinical
Table 2

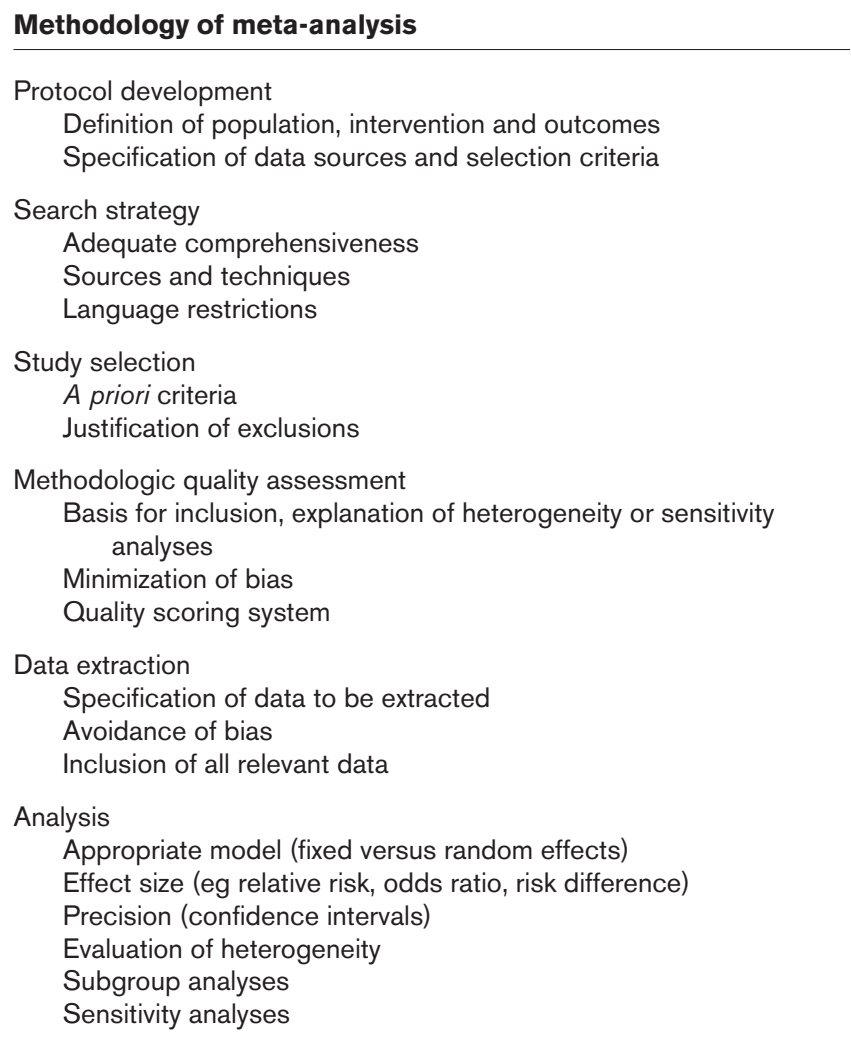

condition of interest. In a model of sepsis, for example, to what extent might the experimental animals conform to the established criteria for human sepsis? Perhaps fitting two or more of the following: temperature $>38$ or $<36^{\circ} \mathrm{C}$; heart rate $>90$ beats $/ \mathrm{min}$; respiratory rate $>20$ breaths $/ \mathrm{min}$ or $\mathrm{PaCO}_{2}<32 \mathrm{mmHg}$; and white blood cells $>12000$ or $<4000$ cells $/ \mathrm{mm}^{3}$, or $>10 \%$ immature (band) forms [30]? Similarly, do the responses of the experimental animals to treatment resemble those anticipated in septic patients? Polymyxin-dextran anti-endotoxin pretreatment in an ovine model of normotensive sepsis produced an array of responses similar to those expected in patients, as shown in Table 3 [31]. This model would thus satisfy at least some of the criteria for appropriate preclinical evaluation of sepsis therapies.

The rigorousness of the study design would serve as another key criterion for evaluating the quality of the proposed research. The already described ovine model study was designed to address the hypothesis that, in sheep with sepsis induced by caecal ligation and perforation, pretreatment with polymyxin-dextran anti-endotoxin would be associated with fewer changes in microvascular integrity and cellular architecture in extrapulmonary organs [31]. The study was, importantly, a prospective doubleblind, randomized trial, thus clearly conforming to the design requirements for definitive clinical trials. 
Table 3

\section{Results of anti-endotoxin pretreatment in an ovine model of normotensive sepsis* [31]}

Less pyrexia $(P=0.04)$

Higher mean arterial pressures $(P=0.02)$

Less variable serum albumin concentration $(P=0.05)$

Trend toward decreased lactate concentration $(P=0.10)$

*48 $\mathrm{h}$ after randomization

The ovine model of sepsis has also been used to compare resuscitation fluids. This blinded study tested the hypothesis that type of fluid infused will modify both microvascular integrity and cellular structure in sepsis [32]. Sheep were randomized to receive crystalloid (Ringer's lactate) or colloid (pentastarch) for $48 \mathrm{~h}$ beginning $6 \mathrm{~h}$ after induction of experimental sepsis. Fluid was infused to maintain constant left atrial pressure. In order to maintain similar plasma volumes (Figure 2), it was necessary to infuse a significantly greater volume of crystalloid than colloid. Higher colloid osmotic pressure was achieved in the colloid recipients (Figure 2). These observations in the ovine model are consistent with those expected in patients. Moreover, fluid was titrated to achieve a target end diastolic filling pressure, and this mode of administration is typical of the fluid management approach taken in the clinical setting. The data in Figure 2 also reveal that the randomized groups were comparable in baseline plasma volume and colloid osmotic pressure. Demonstration of the comparability of the study groups at baseline is also important in randomized clinical trials. While both groups attained a similar hyperdynamic circulatory response, the pentastarch group exhibited greater capillary luminal areas with less endothelial swelling and less parenchymal injury. This blinded randomized trial in an animal model thus provided evidence of benefit with a colloid used in a manner similar to that employed in patients.

Another case study involving blood or blood-substitute preparations demonstrates the application of animal studies in elucidating underlying physiologic mechanisms of clinical relevance. It has been shown that transfusion of older blood was associated with evidence of gastric mucosal ischaemia in septic patients [33]. Red cell transfusion was also found ineffective for increasing splanchnic tissue oxygen utilization in septic patients as measured by gastric $\mathrm{pHi}$ [34]. These observations suggested that the age of transfused blood might be an important determinant of tissue oxygenation, and this possibility was assessed in a rodent model. Storage of rat red blood cells in this prospective randomized trial for $\mathbf{2 8}$ days impaired the ability to improve tissue oxygenation when transfused into either control or septic rats in a state of systemic oxygen uptake $\left(\mathrm{VO}_{2}\right)$ supply dependency induced by iso-

\section{Figure 2}

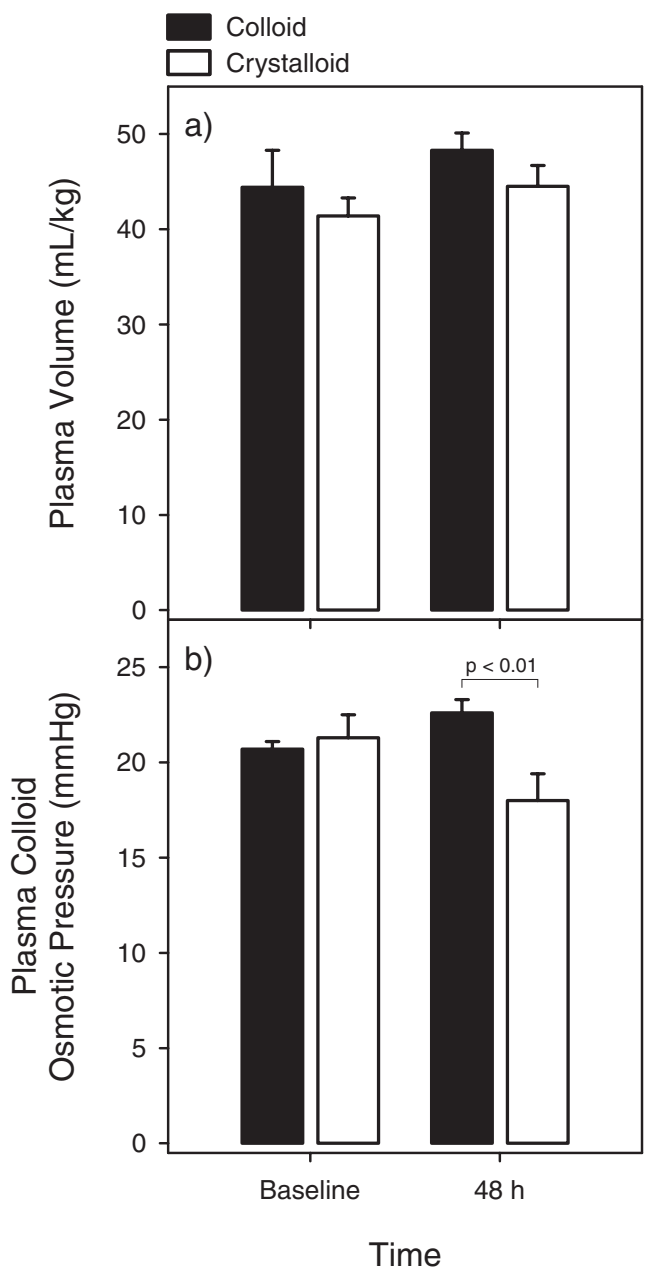

Effects of fluids in an ovine model of sepsis. (a) Plasma volume and (b) plasma osmotic pressure at baseline and $48 \mathrm{~h}$ in sheep with experimental sepsis receiving either pentastarch (black bars) or Ringer's lactate (white bars). Based on the data of Morisaki et al [32].

volaemic haemodilution [35]. Similar observations have subsequently also been made in an ovine model.

The impaired oxygenation associated with stored blood may be a temporary phenomenon, since these blood cells may eventually undergo rejuvenation. Intravital video microscopy observations have indicated the occurrence of capillary dropout after transfusion of stored blood. Such dropout might be due to the increased rigidity of the stored cells, as well as, possibly, increased adhesion to endothelium. It is clear, in any case, that rigorously designed animal studies can have an important role to play in deducing physiologic mechanisms relevant to clinical fluid management. 


\section{Conclusion}

The fluid management landscape has been altered by recent meta-analyses, whatever their methodological limitations may have been. Yet important questions regarding optimal fluid management remain unanswered, and more research is needed. Experimental models that mimic, as faithfully as possible, a clinical condition of interest can make an important contribution. An alternative pathway of preclinical research might serve to reduce the risks and costs of developing therapies, thereby benefiting all participants in the healthcare system. Key elements of this pathway consist of hypotheses specified by developers of therapeutics, a competitive research process, randomized studies in clinically relevant animal models and critical appraisal, possibly including meta-analysis of animal data.

\section{Commentary}

Jean-Louis Vincent, MD, PhD: Are not the data already reported on albumin administration in preclinical models sufficient? Much evidence has accumulated from the experimental studies of colloids as compared with crystalloids. What are the animal studies remaining to be done?

William J Sibbald, MD: That question can only be answered when framed very specifically in terms of the clinical condition of interest and the intended therapeutic intervention. In this context, it is not at all clear that all the relevant studies have already been performed.

Andrew $R$ Webb, MD: Also, the quality of the available data should be considered. Much of what has been reported regarding colloids and crystalloids cannot be readily interpreted in terms of implications for clinical practice.

Jean-Louis Vincent, MD, PhD: Are there not also important limitations to the clinical questions that can be meaningfully addressed in animal models? For example, models of multiple organ failure are difficult to devise. Also, adult respiratory distress syndrome cannot easily be reproduced in animals.

William J Sibbald, MD: While there may be some limitations, the versatility of animal models in mimicking a broad range of clinical conditions should nevertheless be recognized.

\section{References}

1. Schierhout G, Roberts I: Fluid resuscitation with colloid or crystalloid solutions in critically ill patients: a systematic review of randomised trials. BMJ 1998, 316:961-964.

2. Cochrane Injuries Group Albumin Reviewers: Human albumin administration in critically ill patients: systematic review of randomised controlled trials. BMJ 1998, 317:235-240.

3. Choi PT, Yip G, Quinonez LG, Cook DJ: Crystalloids vs. colloids in fluid resuscitation: a systematic review. Crit Care Med 1999, 27: 200-210.

4. Roberts I, Edwards P, McLelland B: More on albumin. Use of human albumin in UK fell substantially when systematic review was published. BMJ 1999, 318:1214-1215.
5. Deitch EA: Animal models of sepsis and shock: a review and lessons learned. Shock 1998, 9:1-11.

6. Michie HR: The value of animal models in the development of new drugs for the treatment of the sepsis syndrome. J Antimicrob Chemother 1998, 41 (suppl A):47-49.

7. Mathiak G, Szewczyk D, Abdullah F, et al: An improved clinically relevant sepsis model in the conscious rat. Crit Care Med 2000, 28: 1947-1952.

8. Abraham $\mathrm{E}$, Wunderink $\mathrm{R}$, Silverman $\mathrm{H}$, et al: Efficacy and safety of monoclonal antibody to human tumor necrosis factor $\alpha$ in patients with sepsis syndrome. A randomized, controlled, double-blind, multicenter clinical trial. JAMA 1995, 273:934-941.

9. Reinhart K, Wiegand-Lohnert C, Grimminger F, et al: Assessment of the safety and efficacy of the monoclonal anti-tumor necrosis factor antibody-fragment, MAK 195F, in patients with sepsis and septic shock: a multicenter, randomized, placebo-controlled, dose-ranging study. Crit Care Med 1996, 24:733-742.

10. Teng NN, Kaplan HS, Hebert JM, et al: Protection against gram-negative bacteremia and endotoxemia with human monoclonal IgM antibodies. Proc Natl Acad Sci USA 1985, 82:1790-1794.

11. Ziegler EJ, Fisher CJ Jr, Sprung CL, et al: Treatment of gram-negative bacteremia and septic shock with HA-1A human monoclonal antibody against endotoxin. A randomized, double-blind, placebocontrolled trial. N Engl J Med 1991, 324:429-436.

12. Quezado ZM, Natanson C, Alling DW, et al: A controlled trial of HA$1 \mathrm{~A}$ in a canine model of gram-negative septic shock. JAMA 1993, 269:2221-2227.

13. McCloskey RV, Straube RC, Sanders C, et al: Treatment of septic shock with human monoclonal antibody HA-1A. A randomized, double-blind, placebo-controlled trial. Ann Intern Med 1994, 121: $1-5$.

14. Rennick $D M$, Fort $M M$ : Lessons from genetically engineered animal models. XII. IL-10-deficient (IL-10(-/-)) mice and intestinal inflammation. Am J Physiol Gastrointest Liver Physiol 2000, 278:G829G833.

15. Piper RD, Cook DJ, Bone RC, Sibbald WJ: Introducing critical appraisal to studies of animal models investigating novel therapies in sepsis. Crit Care Med 1996, 24:2059-2070.

16. Drews J, Ryser S: The role of innovation in drug development. Nat Biotechnol 1997, 15:1318-1319.

17. Cook DJ, Sackett DL, Spitzer WO: Methodologic guidelines for systematic reviews of randomized control trials in health care from the Potsdam Consultation on Meta-Analysis. J Clin Epidemiol 1995, 48:167-171.

18. Stroup DF, Berlin JA, Morton SC, et al: Meta-analysis of observational studies in epidemiology: a proposal for reporting. JAMA 2000, 283:2008-2012.

19. Freedman LS: Meta-analysis of animal experiments on dietary fat intake and mammary tumours. Stat Med 1994, 13:709-718.

20. Freedman LS, Clifford CK: Meta-analysis of animal experiments: elucidating relationships between dietary fat and mammary tumor development in rodents. Adv Exp Med Biol 1994, 364:93-100.

21. McKnight $\mathrm{B}$ : Considerations in the conduct of meta-analysis using data from animal carcinogenicity experiments. IARC Sci Publ 1992, 116:557-569.

22. Valberg PA, Crouch EA: Meta-analysis of rat lung tumors from lifetime inhalation of diesel exhaust. Environ Health Perspect 1999, 107:693-699.

23. Perna A, Remuzzi G: Abnormal permeability to proteins and glomerular lesions: a meta-analysis of experimental and human studies. Am J Kidney Dis 1996, 27:34-41.

24. Benignus VA, Boyes WK, Bushnell PJ: A dosimetric analysis of behavioral effects of acute toluene exposure in rats and humans. Toxicol Sci 1998, 43:186-195.

25. Craig JC, Wheeler DM, Irwig L, Howman-Giles RB: How accurate is dimercaptosuccinic acid scintigraphy for the diagnosis of acute pyelonephritis? A meta-analysis of experimental studies. J Nucl Med 2000, 41:986-993.

26. Bardo MT, Rowlett JK, Harris MJ: Conditioned place preference using opiate and stimulant drugs: a meta-analysis. Neurosci Biobehav Rev 1995, 19:39-51.

27. Kelley G: Mechanical overload and skeletal muscle fiber hyperplasia: a meta-analysis. J App/ Physiol 1996, 81:1584-1588.

28. Linde $\mathrm{K}$, Jonas WB, Melchart $\mathrm{D}$, et al: Critical review and metaanalysis of serial agitated dilutions in experimental toxicology. Hum Exp Toxicol 1994, 13:481-492. 
29. Wilson MA, Chou MC, Spain DA, et al: Fluid resuscitation attenuates early cytokine mRNA expression after peritonitis. I Trauma 1996, 41:622-627.

30. Bone RC, Sibbald WJ, Sprung CL: The ACCP-SCCM consensus conference on sepsis and organ failure. Chest 1992, 101: $1481-1483$.

31. Doig GS, Martin CM, Sibbald WJ: Polymyxin-dextran antiendotoxin pretreatment in an ovine model of normotensive sepsis. Crit Care Med 1997, 25:1956-1961.

32. Morisaki H, Bloos F, Keys J, et al: Compared with crystalloid, colloid therapy slows progression of extrapulmonary tissue injury in septic sheep. J Appl Physiol 1994, 77:1507-1518.

33. Marik PE, Sibbald WJ: Effect of stored-blood transfusion on oxygen delivery in patients with sepsis. JAMA 1993, 269:3024-3029.

34. Silverman HJ, Tuma P: Gastric tonometry in patients with sepsis. Effects of dobutamine infusions and packed red blood cell transfusions. Chest 1992, 102:184-188.

35. Fitzgerald RD, Martin CM, Dietz GE, et al: Transfusing red blood cells stored in citrate phosphate dextrose adenine-1 for 28 days fails to improve tissue oxygenation in rats. Crit Care Med 1997, 25:726-732.

Author's affiliation: Department of Medicine, Sunnybrook and Women's College Health Sciences Centre, Toronto, Ontario, Canada

Correspondence: William J Sibbald, MD, FRCPC, CHE, Physician in Chief, Department of Medicine, Room D474, Sunnybrook and Women's College Health Sciences Centre, University of Toronto, 2075 Bayview Avenue, Toronto, Ontario, Canada M4N 3M5.

Tel: +1 416480 4592; fax: +1 4164806191 ;

e-mail:William.Sibbald@swchsc.on.ca 